\title{
A CHART FOR PLOTTING RELATIONS BETWEEN VARIABLES OVER THEIR ENTIRE REAL RANGE*
}

\author{
By L. H. DONNELL (Illinois Institute of Technology)
}

The following simple method of graphical respresentation, covering the entire real range, seems rather obvious and the writer has found it useful for many years; however he has never seen it described in the literature. It consists of an ordinary Car-

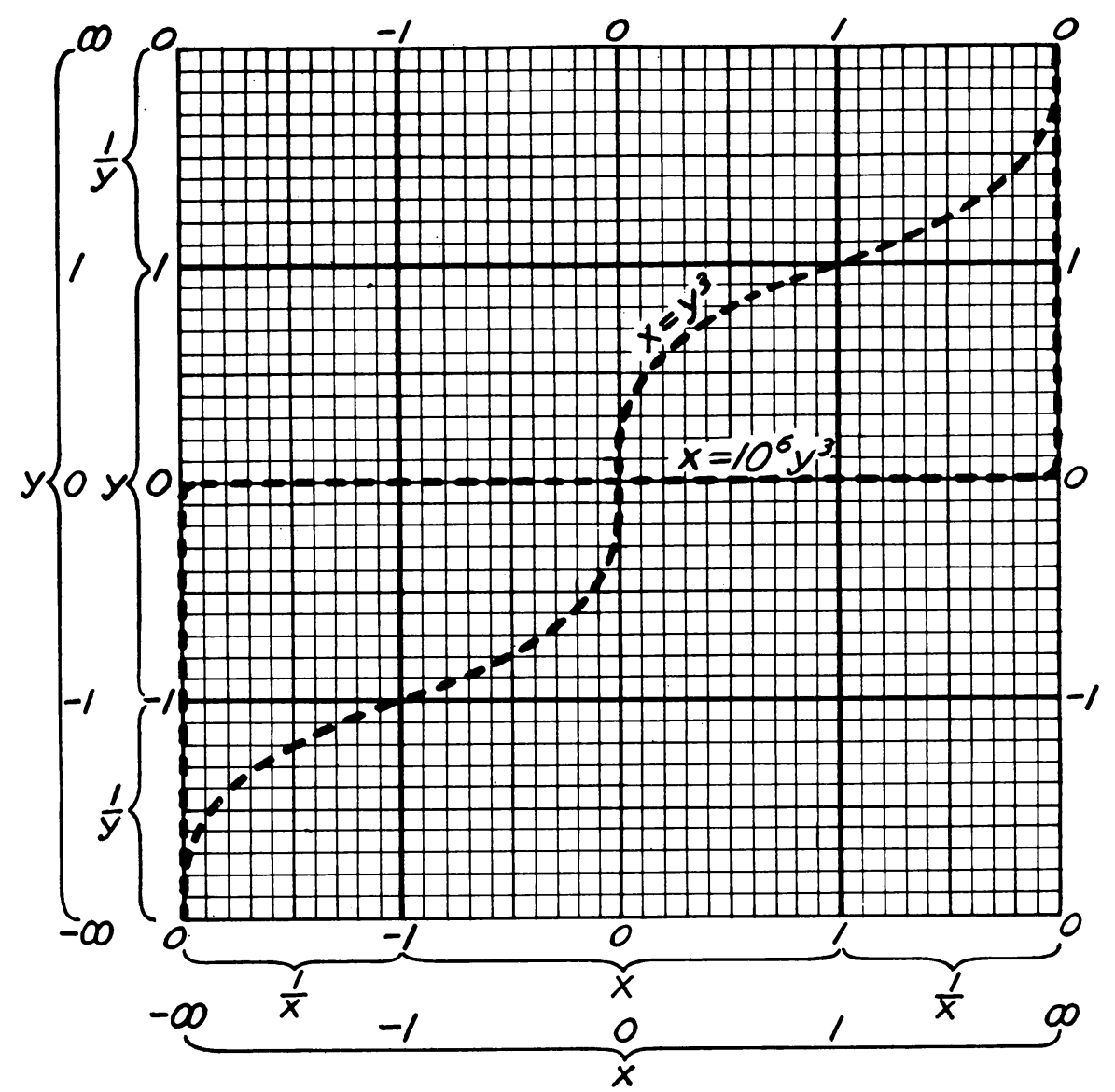

FIG. 1.

tesian plot over the range -1 to +1 of each variable, with adjoining Cartesian plots of the reciprocals of the variables from 0 to -1 and from +1 to 0 , arranged as shown in Fig. 1. This is evidently equivalent to a plot over the range $-\infty$ to $+\infty$ for each variable. It is also evident that curves and their slopes will be continuous over the dividing lines between the two kinds of plots if the functions represented and their first derivatives are continuous at these points.

* Received March 22, 1943. 
To illustrate the method, the power relation $x=y^{n}$ has been plotted in Fig. 2 for values of $x$ and $y$ between $-\infty$ and $+\infty$, and for various values of $n$.

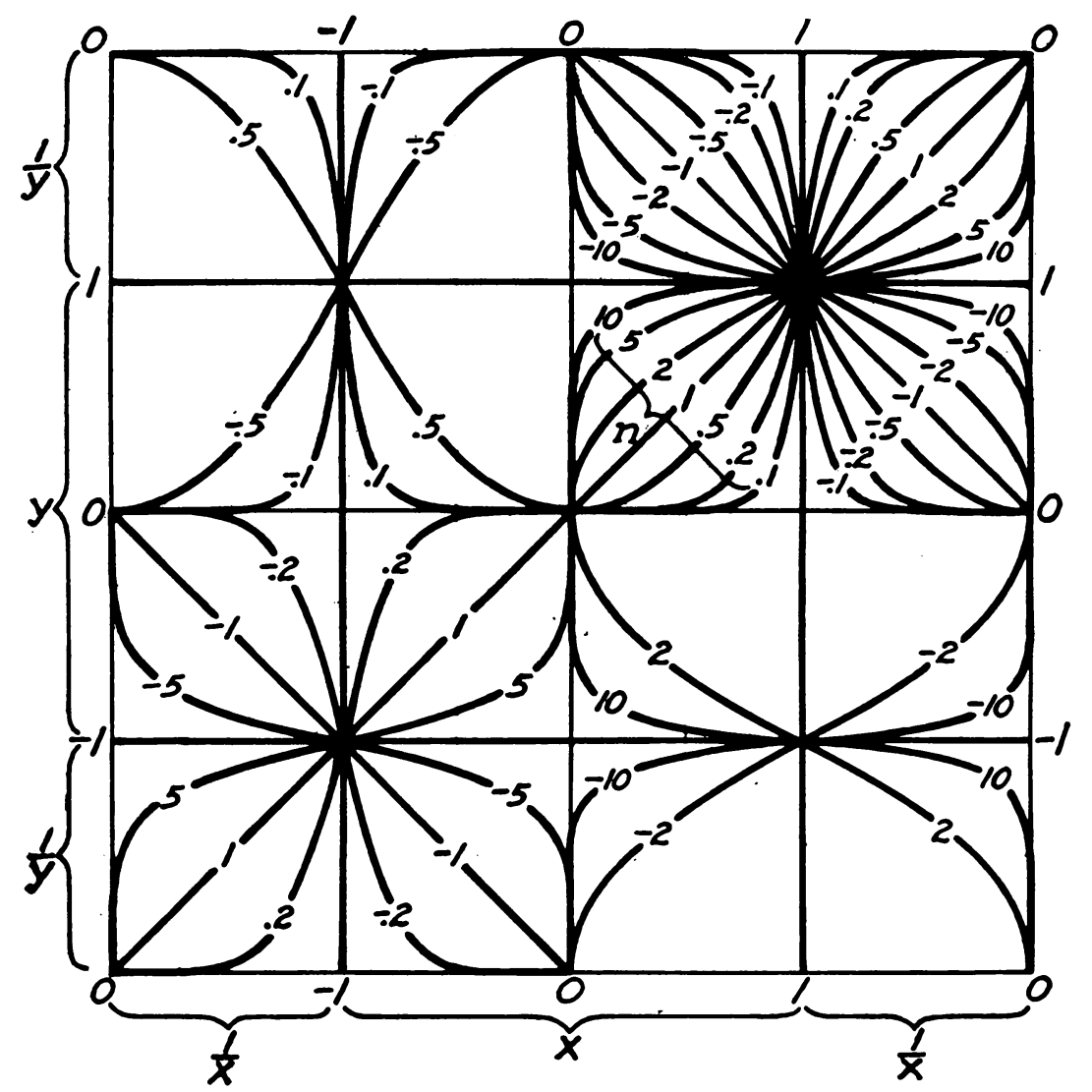

FIG. 2. Plot of $x=y^{n}$.

In many applications only a part of such a chart would be required. For instance only the upper right hand quarter would be needed to cover all positive real values of the variables.

In some cases a change of variable will make the resulting plot much more useful. For instance a plot of the relation $x=10^{6} y^{3}$, (see Fig. 1) follows the coordinate axes closely and would be nearly indistinguishable from many other functions, such as $x=10^{10} y^{3}$ or $x=10^{10} y^{5}$. On the other hand, a plot of $x=y^{\prime 3}$, where $y^{\prime}=100 y$, gives a much more illuminating and characteristic picture of the function. Any change of variable which makes the resulting curve pass through or near such points as $(1,1)$, $(1,-1),(-1,1)$ or $(-1,-1)$ would accomplish this purpose in most cases. 